\title{
Transmission of mitochondrial DNA - playing favorites?
}

\author{
Jeffrey L. Boore
}

\begin{abstract}
Summary
Mitochondria are essential subcellular organelles containing an extranuclear genome (mtDNA). Mutations in mtDNA have recently been identified as causing a variety of human hereditary diseases. In most of these cases, the tissues of the affected individual contain a mixture of mutant and normal mtDNA, with this ratio determining the severity of symptoms. Stochastic factors alone have generally been believed to determine this ratio. Jenuth et al.(1), however, examining mice that contain a mixture of mtDNA types, show evidence of strong selective forces at work in favoring one mtDNA variant over another in some tissues.
\end{abstract}

\section{Mitochondrial DNA transmission}

Mammalian mitochondrial DNA (mtDNA) is a maternally inherited, circular genome of approximately $16.6 \mathrm{~kb}$. It contains 37 genes encoding 13 protein subunits of the enzymes involved in oxidative phosphorylation and the two rRNAs and 22 tRNAs necessary for translation of these proteins. Mitochondria have been best studied as producers of ATP, but they also perform a myriad of other functions, including the production of heme, lipids, amino acids and pyrimidines, and the regulation of inorganic ions ${ }^{(2)}$. The diverse functions of mitochondria require many additional proteins and other elements, all of which are imported products of nuclear genes. Oddly, there are even recent suggestions that the role of mtDNA may not be limited to its subcellular compartment - for example, the mitochondrially encoded ND1 protein of mice appears to be (inexplicably) a cell surface antigen ${ }^{(3)}$, and the mitochondrial large subunit rRNA is found to play a role in Drosophila embryogenesis as a component of the cytoplasm ${ }^{(4,5)}$.

The rate of nucleotide substitution in mtDNA is 5-10 times higher than in nuclear genes ${ }^{(6)}$, leading to a high level of polymorphism within populations. However (notable exceptions notwithstanding; see refs 7-9), various tissues of the same individual typically share only one form, a condition referred to as homoplasmy. This seeming incongruence is explained by two factors. Firstly, paternal mitochondria seldom enter the egg at fertilization and those that do are actively targeted for destruction ${ }^{(10)}$. Therefore, heteroplasmy is not due to a combination of differing $m t D N A s$ from two parents; mtDNA variation can only build clonally through maternal lines. Secondly, the population of mtDNA molecules passes through a bottleneck in the germline at each generation. While a mature oocyte has $\sim 100,000$ mitochondria, only a small number $(11,12)$ become localized to the primordial germ plasm $(13,14)$. Only this subset will then contribute to the mtDNA population of all of the egg cells of the individual resulting from this fertilization. The result is that stochastic factors drive out variation rather quickly. This is seen in a four-generation study of Holstein cows, which showed that heteroplasmy level shifts substantially in a single generation, that a return to homoplasmy occurs within only two or three generations, and that neutral variants of mtDNA are seen to segregate in different directions in two siblings ${ }^{(11)}$.

Similarly, distribution of any mtDNA variation in the zygote among somatic tissues of the developing embryo can be governed by stochastic factors. Any mutant mtDNAs that are present in the primordial germ plasm expand in number along with normal copies to populate the oocyte for the next generation. The result of fertilization is a chimera of mtDNA haplotypes, with forces analogous to genetic drift allocating them among the tissues of the animal. The same mathematical models estimate the probability of a rare variant becoming fixed as being the same as its original frequency in the population ${ }^{(15)}$. In some cases, this has been shown experimentally. Jenuth et al.(1) sampled individual colonic crypts from mice that had been engineered to begin life with $92 \%$ of a mtDNA variant (see below). The stem cells that produce these crypts divide about every 24 hours, and so about $92 \%$ of the crypts should be fixed for this variant in 15-month-old mice; in fact, $93 \%$ of the colonic crypts were fixed for the introduced mtDNA.

Under some circumstances, however, a radical departure from this model of random distribution of mtDNA variants is seen. Tissues or cells appear to be selecting one mtDNA variant over another. What are the processes that determine the actual distribution of mtDNA variants, pathological or neutral, among the tissues as an animal ages? As discussed below, the answer is important for understanding the 
progression of several degenerative human diseases and perhaps for understanding aging itself. Studies are only beginning to address the nature of these factors.

\section{Role of mtDNA heteroplasmy in human pathology}

Deletions, duplications and point mutations in mtDNA have each been identified as the cause of several human hereditary diseases (reviewed in ref. 16). One example, KearnsSayre syndrome, is a devastating disease caused by a largescale deletion of mtDNA, often of the order of $5-7 \mathrm{~kb}^{(17)}$. Chen et al. ${ }^{(18)}$ used quantitative PCR to show that identical deletions are present in normal human oocytes at a frequency as high as $0.1 \%$. This presumably reflects the spontaneous deletion rate because it would be unlikely that this low level would persist through the 'bottleneck'. Perhaps the disease is inherited only when a deleted mtDNA comes to predominate the subset destined for the primordial germ plasm. Does chance alone determine this, or could there be some mechanism selecting deleted mtDNAs for greater representation?

Other examples of hereditary disease caused by mtDNA deficiency include LHON (Leber's hereditary optical neuropathy) ${ }^{(19)}$, NARP (neurogenic muscle weakness ataxia and retinitis pigmentosa) ${ }^{(20)}$ and MELAS (mitochondrial encephalomyopathy, lactic acidosis and stroke-like episodes)(21). Scores of other human mitochondrial mutations have been identified as causing pathologies ranging from mild to lethal(16). In each of these cases, the tissues of the affected individual contain a mixture of mutant and normal mtDNA. This ratio often changes over the life of the individual and the percentage of mutant mtDNA is generally correlated with the severity of symptoms. Since mtDNA mutations seem to rapidly segregate to homoplasmy, usually each case of disease is thought to result from a de novo mutation. Tissues vary in their sensitivity to mitochondrial function deficiency; generally brain is the most sensitive, followed by heart, muscle, kidney and endocrine systems. Although critical for diagnosing and predicting the course of these diseases, the factors determining the distribution of normal and mutant mtDNAs within the tissues of an individual are still poorly understood.

The tissue distribution of defective mtDNA molecules may also be critical to the aging process. There is growing evidence that the general decline in aerobic capacity accompanying aging is largely the result of accumulated mutant mtDNA molecules in somatic tissues ${ }^{(16)}$. MtDNA molecules are damaged by their regular exposure to the reactive oxygen species produced by oxidative phosphorylation such as $\mathrm{H}_{2} \mathrm{O}_{2}, \mathrm{OH}$ and $\mathrm{O} \cdot \overline{2}^{(22)}$. Disease caused by the inheritance of defective mtDNA molecules is generally progressive; it may be that this is due to the normal accumulation of defective mtDNAs in tissues already challenged with a high percentage of defective mitochondria.

\section{Mouse cybrid experiments}

Jenuth et al.(1) constructed cybrid (cytoplasmic hybrid) mouse embryos by electrofusing donor cytoplasts (cytoplasm bound in membrane) to recipient one-cell embryos. This created heteroplasmic mice carrying two different mitochondrial haplotypes. They refer to these as the 'BALB' and 'NZB' haplotypes, which are known to vary at $106 \mathrm{nts}(0.6 \%$ of the mtDNA) ${ }^{(3)}$ scattered throughout the genome. They then compared the distribution of these two mtDNA haplotypes in several tissues at various ages (Fig. 1). Using NZB mtDNA as the donor haplotype, the constancy in newborns' tissues seems remarkable (3.2-5.3\%), suggesting little or no selection on mtDNA haplotype during fetal development. However, by 4 months of age, a significant shift has occurred. Blood and spleen have greater amounts of the BALB haplotype, kidney and liver of the NZB haplotype, while other tissues remain at about $4 \%$ NZB. This same effect was observed in all of the 37 animals studied. By 12 months of age these effects are even more pronounced, with the NZB haplotype representing as high as $53.2 \%$ of the mtDNA in liver. By using a population genetics model for selection in asexual populations and assuming a 67-day turnover time of mtDNA in hepatocytes $^{(23)}$, they calculate that the NZB haplotype has a $50 \%$ selective advantage at each cell generation to account for this increase from $3.3 \%$ to $53.2 \%$ representation.

What is responsible for this radical departure from random drift in the fixation of these mtDNA haplotypes? Selection could be at any of three levels: one mtDNA haplotype may have a replication advantage; differing mitochondria may change their numbers within a cell; and differing cells may be selected within a tissue. The differences between these two haplotypes which can be recognized consist of only 106 isolated nucleotide substitutions, 15 of which lead to amino acid replacement. If there is a replication advantage conferred by these nucleotide substitutions, it falls outside of the conserved sequences so far recognized as controlling elements ${ }^{(24,25)}$. Perhaps cells can signal certain

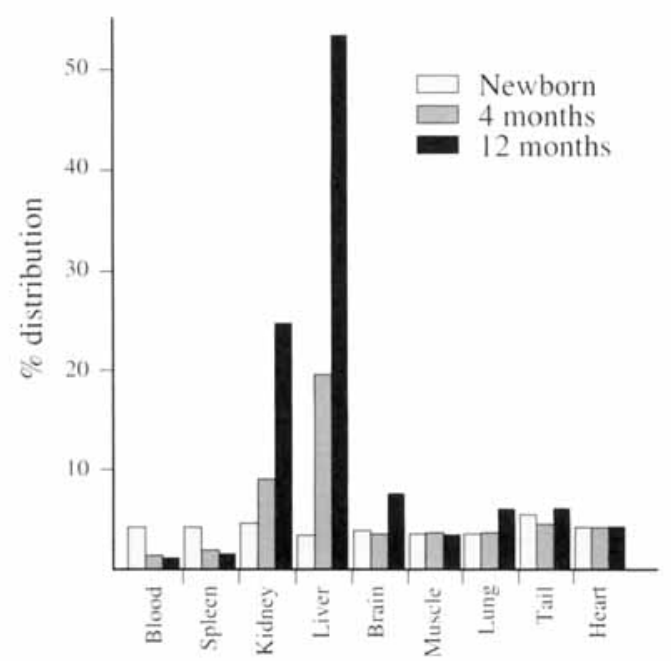

Fig. 1. Tissue-specific distribution of the NZB mtDNA genotype (the donor genotype) in cybrid mice of various ages $(1)$. 
mitochondria for more active biogenesis, possibly as a negative feedback loop for poor functioning; if so, the variations in coding sequence could cause a difference in mitochondrial functioning that might lead to increased representation of one haplotype over the other. Finally, cells containing a particular mtDNA variant may have a tissue-specific growth advantage leading to selection for mtDNA haplotype at the level of the cell. Tissue-specific isoforms of nuclearencoded mitochondrial proteins have been identified, suggesting a candidate mechanism ${ }^{(26)}$.

\section{Studies in other systems}

At least three other recent studies have also addressed the question of how mtDNA is distributed among somatic tissues. Mitochondria from patients with MELAS along with wild-type mitochondria have been introduced into two human mitochondrially deficient cell lines. Clonal lines generated show strong selection for one or the other haplotype, depending on the nuclear background(27). Mathews et al.(28) compared the tissue distribution of the MELAS-causing point mutation at position 3243 in several related adults and one fetus. The ratio of mutant to normal mtDNAs varied widely among the same tissues of different adults and among the tissues of the same adult (3-67\%). However, six tissues of the fetus had a mean percentage of mutant mtDNA of $53 \%$ with a standard deviation of only $2 \%$, suggesting that additional factors act to change the tissuespecific ratios after birth. Cytoplasmic injection between Drosophila eggs carrying different mtDNA haplotypes show one favored by $58 \%$ per fly generation ${ }^{(29)}$, although the amount and persistence of heteroplasmy found in this study suggests that there may be significant differences between fly and mammalian systems.

One interesting related topic - while somatic tissues accumulate a measurable amount of mtDNA mutants even in normal individuals ${ }^{(30)}$, the germ line must somehow evade this process. Presumably this is accomplished by sequestering a subset of mitochondria into primordial germ plasm. Is this a 'safer' environment? Do these mitochondria generate fewer free radicals to minimize DNA damage? Are these mitochondria active during the long period of suspended prophase or is there subdivision between the 'working' mitochondria and those protected for the good of their genes? Could these mtDNAs be enzymatically protected in some way or is it simply enough that they undergo fewer rounds of replication? Important issues in population genetics, medicine, and molecular and developmental biology are addressed by our understanding of this system.

\section{References}

1 Jenuth, J., Peterson, A. and Shoubridge, E. (1997). Tissue-specific selection for different mtDNA genotypes in heteroplasmic mice. Nature Genetics 16, 93-95. 2 Tzagoloff, A. (1982). Mitochondria. Plenum, New York.

3 Loveland, B., Wang, C.-R., Yonekawa, H., Hermel, E. and Lindahl, K. (1990). Maternally transmitted histocompatibility antigen of mice: A hydrophobic peptide of a mitochondrially encoded protein. Cell60,971-980.
4 Kobayashi, S. and Okada, M. (1989). Restoring of pole cell-forming ability to u.v. irradiated Drosophila embryos by injection of mitochondrial IrRNA. Development 107, 733-742

5 Kobayashi, S., Amikura, R. and Okada, M. (1993). Presence of mitochondrial large ribosomal RNA outside mitochondria in germ plasm of Drosophila melanogaster. Science 260, 1521-1524.

6 Brown, W. M. (1985). The mitochondrial genome of animals. In Molecular Evolutionary Genetics (ed. R. J. Macintyre), pp. 95-130. Plenum Press, New York. 7 Bendall, K. and Sykes, B. (1995). Length heteroplasmy in the first hypervariable segment of the human miDNA control region. Am. J. Human Genet. 57, 248-256.

8 Magoulas, A. and E. Zouros. (1991). Restriction-site heteroplasmy in anchovy (Engraulis encrasicolus) indicates incidental biparental inheritance of mitochondrial DNA. Mol. Biol. Evol. 10, 319-325.

9 Stewart, D., Kenchington, E., Singh, R. and Zouros, E. (1996). Degree of selective constraint as an explanation of the different rates of evolution of genderspecific mitochondrial DNA lineages in the mussel Mytilus. Genetics 143, 13491357.

10 Kaneda, H., Hayashi, J.-I., Takahama, S., Taya, C., Lindahl, K, and Yonekawa, H. (1995). Elimination of paternal mitochondrial DNA in intraspecific crosses during early mouse embryogenesis. Proc. Natl Acad. Sci. USA 92, 4542 4546

11 Ashley, M., Laipis, P and Hauswirth, W. (1989). Rapid segregation of heteroplasmic bovine mitochondria. Nucl. Acids Res. 17, 7325-7331.

12 Jenuth, J., Peterson, A., Fu, K. and Shoubridge, E. (1996). Random genetic drift in the female germline explains the rapid segregation of mammalian mitochondrial DNA. Nature Genetics 14, 146-151.

13 Mignotte, F., Tourte, M. and Mounolou, J.-C. (1987). Segregation of mitochondria in the cytoplasm of Xenopus vitellogenic oocytes. Biol. Cell 60, 97 . 102

14 Akiyama, T. and Okada, M. (1992). Spatial and developmental changes in the respiratory activity of mitochondria in early Drosophila embryos Development 115, 1175-1182

15 Birky, C. W. (1983). Relaxed cellular controls and organelle heredity. Science 222, 468-475

16 Wallace, D. (1995). Mitochondrial DNA variation in human evolution. degenerative disease, and aging. Am. J. Hum. Genet. 57, 201-223.

17 Moraes, C. et al. (1989). Mitochondrial DNA deletions in progressive external ophthalmoplegia and Kearns-Sayre syndrome. New Engl. J. Med. 320, 12931299

18 Chen, X., Prosser, R., Simonetti, S., Sadlock, J., Jagiello, G. and Schon E. (1995). Rearranged mitochondrial genomes are present in human oocytes. Am. J. Hum. Genet. 57, 239-247.

19 Wallace, D. et al. (1988). Mitochondrial DNA mutation associated with Leber's hereditary optic neuropathy. Science 242, 1427-1430.

20 Holt, I., Harding, A. Petty, R. and Morgan-Hughs, J. (1990). A new mitochondrial disease associated with mitochondrial DNA heteroplasmy. Am. J Hum. Genet. 46, 428-433.

21 Goto, Y., Nonaka, I. and Horai, S. (1995). A mutation in the tRNA Leu(UUR) gene associated with the MELAS subgroup of mitochondrial encephalomyopathies. Nature 348, 651-653.

22 Richter, C. et al. (1995). Oxidants in mitochondria: from physiology to diseases. Biochim. Biophys. Acta 1271, 67-74.

23 Zajicek, G. Oren, R. and Weinreb, M. (1985). The streaming liver. Liver 5, 293 300.

24 Shadel, G. and Clayton, D. (1993). Mitochondrial transcription initiation. variation and conservation. J. Biol. Chem. 268, 16083-16086.

25 Clayton, D. (1992). Transcription and replication of animal mitochondrial DNAs. Int. Rev. Cytol. 141, 217-232.

26 Kennaway, N. et al. (1990). Isoforms of mammalian cytochrome c oxidase correlation with human cytochrome $c$ oxidase deficiency. Pediatr. Res. 28, 529 535.

27 Dunbar, D., Moonie, P., Jacobs, H. and Holt, I. (1995). Different cellular backgrounds confer a marked advantage to either mutant or wild-type mitochondrial genomes. Proc. Natl Acad. Sci. USA 92, 6562-6566.

28 Mathews, P., Hopkin, J., Brown, R., Stephenson, J., Hiiton-Jones, D. and Brown, G. (1994). Comparison of the relative levels of the 3243 (A-G) mtDNA mutation in heteroplasmic adult and fetal tissues. J. Med. Genet. 31, 41-44.

29 de Stordeur, E., Solignac, M., Monnerot, M. and Mounolou, J.-C. (1989) The generation of transplasmic Drosophila simulans by cytoplasmic injection: Effects of segregation and selection on the perpetuation of mitochondrial DNA heteroplasmy. Mol. Gen. Genet. 220, 127-132.

30 Wallace, D. et al. (1995). Mitochondrial DNA mutations in human degenerative diseases and aging. Biochim. Biophys. Acta 1271, 141-151.

Jeffrey L. Boore is at the Department of Biology, University of Michigan, 830 N. University Ave., Ann Arbor, MI 48109, USA. 